\title{
Equipment Vibration Budget for TMT
}

\author{
Douglas G. MacMartin*,a,b and Hugh Thompson ${ }^{b}$ \\ ${ }^{a}$ Computing + Mathematical Sciences, California Institute of Technology \\ 1200 E. California Blvd., Pasadena, CA 91125 \\ ${ }^{b}$ TMT Observatory Corp., 1111 S. Arroyo Parkway, Suite 200, Pasadena CA 91105
}

\begin{abstract}
Vibration from equipment mounted on the telescope and in summit support buildings has been a source of performance degradation at existing observatories, for adaptive optics performance in particular. To ensure that that the total optical performance degradation due to vibration is less than the corresponding optical error budget allocation, a vibration budget has been created that specifies allowable force levels from each source of vibration in the observatory (e.g., pumps, chillers, cryocoolers, etc.). In addition to its primary purpose, the vibration budget allows us to make design trade-offs, specify isolation requirements for equipment, and tighten or widen individual equipment vibration specifications as necessary.

Defining this budget relies on two types of information: (i) vibration transmission analysis that determines the optical consequences that result from forces applied at different locations in the Observatory and at different frequencies; and (ii) initial estimates for plausible source amplitudes in order to allocate force budgets to different sources in the most realistic and cost-effective manner. The transmission of vibration from sources through to their optical consequences uses the finite element model of the telescope structure, including primary mirror segment models and control loops. Both the image jitter and higher-order deformations due to M1 segment motion are included, along with the spatial- and temporal-correctability by the adaptive optics system. Measurements to support estimates of plausible soil transmissibility are described in a companion paper. As the detailed design progresses and more information is available regarding what is achievable at realistic cost, the vibration budget will be refined.
\end{abstract}

\section{Keywords: Vibration, Extremely Large Telescopes, Performance, Adaptive Optics}

\section{INTRODUCTION}

The Thirty Meter Telescope (TMT) will be significantly larger than existing ground-based optical telescopes, and will deliver unprecedented performance for both adaptive optics (AO) and seeing-limited observations. Vibration due to equipment both on and off the telescope is a source of potential performance degradation, for adaptive optics (AO) observations in particular; see for example the comprehensive review by Kulcsár et al. ${ }^{1}$ For TMT, our error budget allocates $30 \mathrm{~nm}$ for the residual AO-corrected rms wavefront error (WFE) due to all vibration sources, including wavefront errors due to both image jitter and M1 segment motion. This is significantly better than what is typically achieved at existing observatories, and will require careful attention during final design and construction.

To ensure that TMT will deliver acceptable AO image quality despite equipment vibration, we place requirements on source amplitudes in Newtons for every significant potential source of vibration; this in turn will affect design decisions such as locating sources off telescope where possible, selecting well-balanced low-vibration equipment, and defining isolation requirements. Our basic approach is to (i) use the telescope finite element model to evaluate the optical sensitivity to forces applied at different locations and at different frequencies, (ii) use this sensitivity analysis to place requirements on source equipment to ensure that the error budget is met, and (iii) evaluate potential vibration sources to determine what steps (e.g., isolation) would be required to meet these requirements, or alternatively assess whether the $\mathrm{AO}$ error budget allocation to vibration should be increased. We intend to complement this model-based approach with transfer-function measurements made using calibrated sources as the observatory is built; initial measurements at Subaru Observatory are described in [2]. Section 2 describes the modeling of optical sensitivity; preliminary results can also be found in [3].

*Email: macmardg@cds.caltech.edu 
The preliminary vibration budget is given in Table 2 in Section 3. The specification applies to the rms force level in Newtons for each source after passing through a filter that has unit gain from $5-20 \mathrm{~Hz}$, but falls off above and below this frequency range. This allows for higher forces at either higher or lower frequency where the AO performance is less sensitive; the shape of the filter is motivated from the modeling described in the next section.

Note that in many applications, the vibration environment is specified in terms of the power spectrum of acceleration. However, while this is appropriate to determine, for example, isolator requirements for some sensitive device that is mounted in such an environment, it is not sufficient for placing requirements on sources that would ensure that such an acceleration power spectrum can be met.

\section{SENSITIVITY ANALYSIS}

Input locations for vibration considered here include the following:

1. Pier forces (applied uniformly at the base of the azimuth track). Note that all off-telescope sources affect the optical response through motion of the pier; the propagation of forces from the enclosure or facilities building is not included in the Finite Element Model (FEM) but is captured by an estimated attenuation factor anchored from data taken at Subaru Observatory. ${ }^{2}$

2. Azimuth and Elevation cable wraps; these are modeled as torques about drive axes. For consistency with other inputs, the response is given per Newton of force, using the cable-wrap radius to scale the moment arm.

3. Instruments and other sources on the Nasmyth platforms. Input forces have been considered at all instrument mass nodes and on the Nasmyth platform itself; the response is similar and results are shown here only for forces at one instrument (MIRES).

4. Laser/M2 electronics and laser heads; these are mounted on the elevation journal and on a platform attached to the journal respectively. M2 electronics are currently planned to also be mounted to the elevation journal and hence the same sensitivity can be used.

5. Laser launch telescope (LLT) and other sources near M2.

6. M3 electronics, at the base of the M3 tower. M1 electronics boxes are distributed under the primary mirror, but the optical sensitivity is assumed to be similar to the central location where M3 electronics are placed.

7. Elevation axis direct drive motor on the azimuth structure. Note that forces associated with the Azimuth motor affect the system similarly to pier forces.

The last four locations are potential sources of vibration through the use of cooling, which may exert forces from fluid turbulence that may be difficult to isolate. Vibration forces may also enter the structure at mounting brackets for the pipes carrying cooling fluid (the flow may be turbulent or include pressure fluctuations from compressors/pumps); as the routing of these has not been established, the vibration budget uses sensitivities from representative locations in the list above. For each source location (other than cable wrap torques and pier vertical force), the response is calculated for forces in each axis $\left(F_{x}, F_{y}\right.$, and $\left.F_{z}\right)$, and the sensitivities shown in Figure 5 calculated as the rms over all three axes.

Vibration caused by sources such as bearing roughness, torque ripple or other actuator noise within the M2 or M3 subsystems overwhelmingly results in local motion of the respective optical surface rather than vibration propagation through the structure (see Fig. 1). These disturbances apply equal and opposite torques to the telescope structure and the relevant optic; while the former can result in excitation of telescope resonances and motion of other optical surfaces, the resulting image motion is negligible compared with the direct response of the driven surface. There is thus no need to consider these inputs with the full structural model. For TMT, these sources are separately categorized in the error budget as "control noise" and are managed by the relevant subsystem directly.

Forces on the telescope structure and pier result in both image jitter and M1 segment dynamic motion. We predict these effects using two separate models, since different fidelity is required. The image jitter prediction combines the telescope FEM with a linear optical model and the mount control system (which only affects results at low frequencies). The M1 segment motion model additionally includes segment dynamics for all 492 primary mirror segments, including the stiffness and internal resonances of the segment support assembly, and actuator 


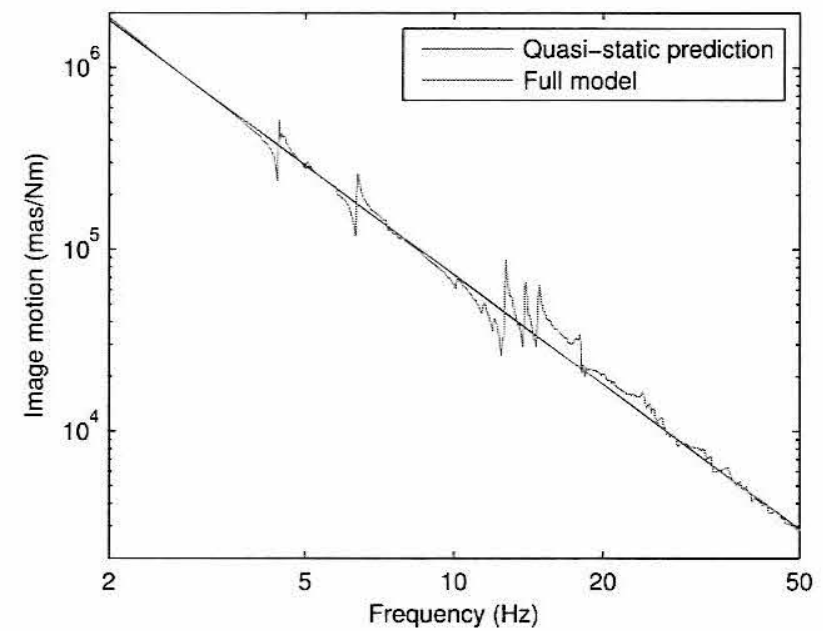

Figure 1. Effect of M2 control noise; the quasi-static rigidbody response due to an applied torque (equal and opposite to M2 and structure) is almost identical to the response computed from the full telescope FEM and optical model, except in narrow bands near a few resonant frequencies.

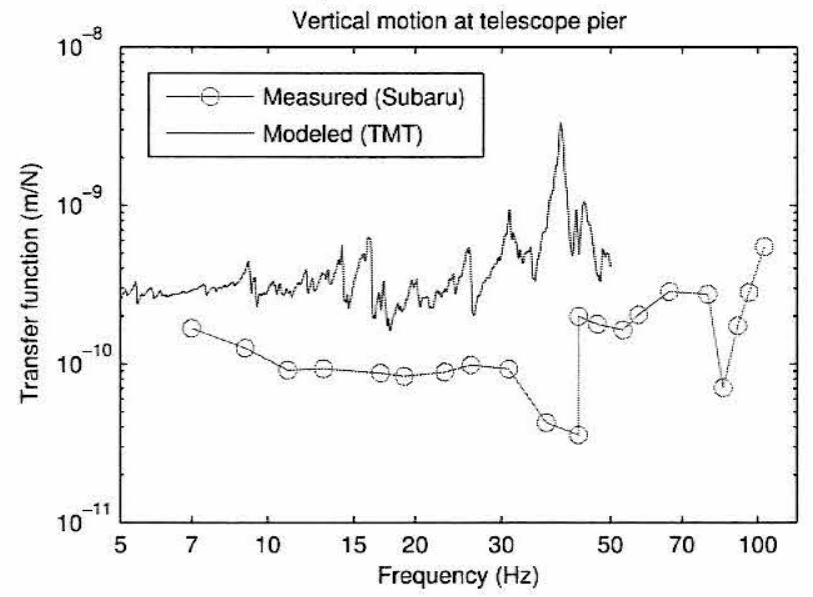

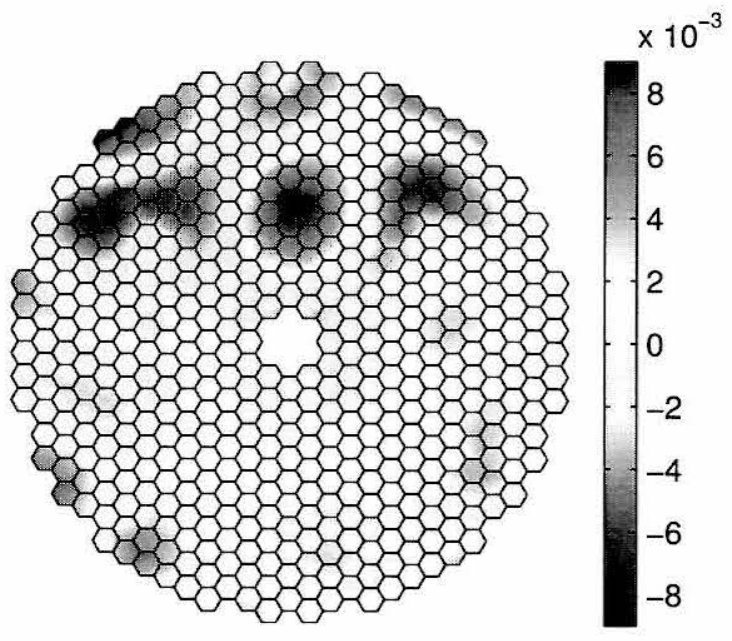

Figure 2. Representative pattern of M1 segment motion at $30 \mathrm{~Hz}$; the resulting wavefront error would be wellcorrected by $\mathrm{AO}$ if it were static, and is limited primarily by the temporal $\mathrm{AO}$ bandwidth.

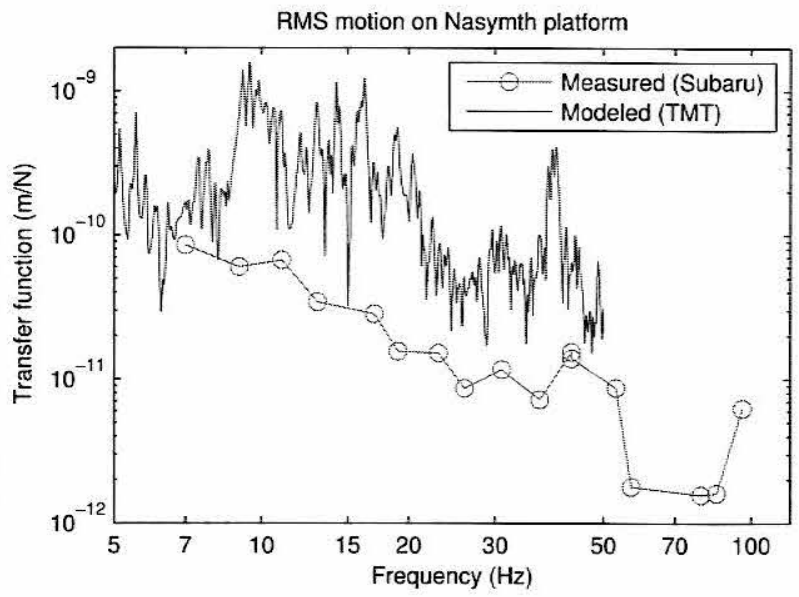

Figure 3. Comparison of measured transfer function (at Subaru ${ }^{2}$ ) with model-based estimate (for TMT) for response at telescope pier (left) and on Nasmyth platform (right). There is no reason for these to be the same, but the roughly comparable levels do provide some confidence in the model-based estimates.

dynamics that include the actuator servo characteristics. Note that TMT has selected (soft) voice-coil actuators for control of M1 segment motion, with stiffness at low frequencies provided by a roughly $8 \mathrm{~Hz}$ servo loop. ${ }^{4}$ Above this frequency, these actuators serve to isolate segment motion from mirror cell motion, greatly reducing the contribution of M1 segment motion to the AO-corrected wavefront error. ${ }^{3}$

In order to build some confidence in the model-based results used to establish the vibration budget, two transfer function predictions made using the TMT model are compared with measured transfer functions collected at Subaru Observatory ${ }^{2}$ in Figure 3. While the telescope structure, pier, and soil stiffness are all different from Subaru, the fact that the response is similar within an order of magnitude improves confidence in the model-based results.

A $15 \mathrm{~Hz}$ Type-II rejection is included for simulating the residual AO-corrected wavefront error from telescope image motion; the relatively low bandwidth results from the requirement of high sky coverage, combined with the need for natural guide stars for tip/tilt estimation. A $60 \mathrm{~Hz}$ bandwidth is used for correction of M1 segment motion by the deformable mirrors of the AO system. At each frequency, the M1 displacement map is computed 

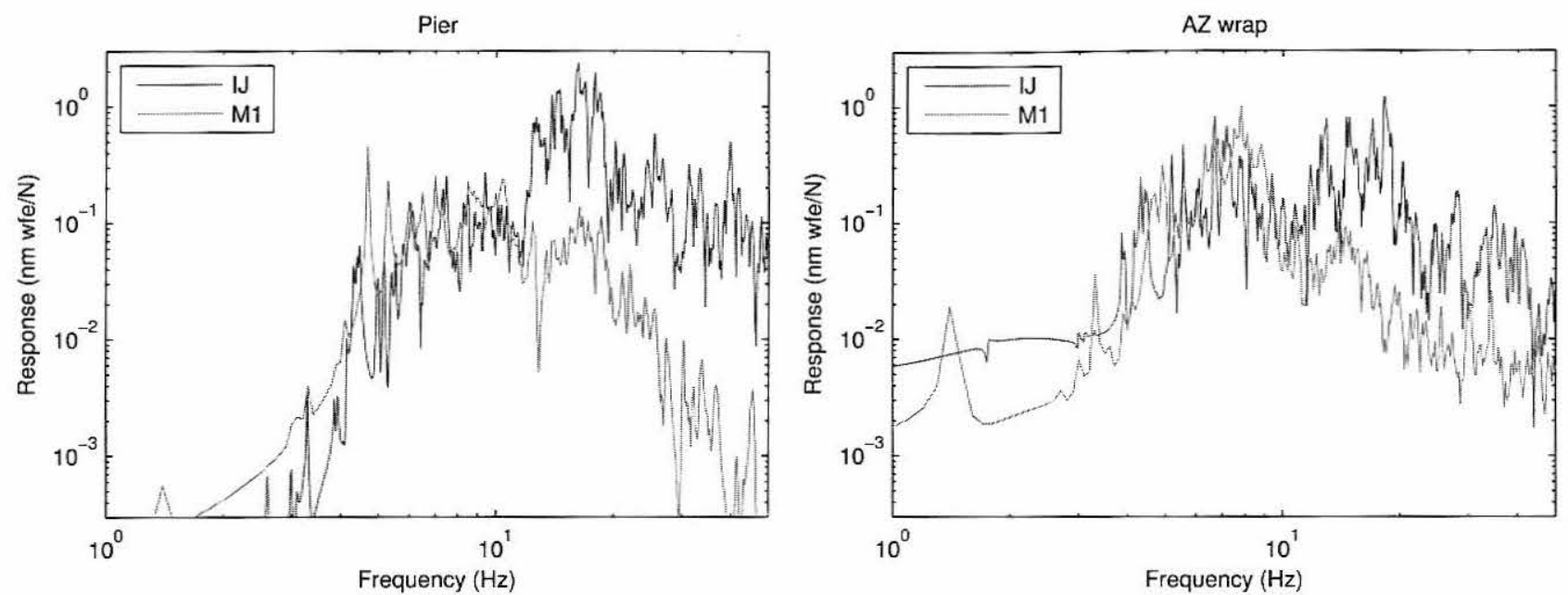

Figure 4. Contribution to residual AO-corrected wavefront error from image motion and from M1 segment motion for force input at the telescope pier (left) and azimuth cable wrap (right). The M1 contribution is typically less important than image jitter above roughly $10 \mathrm{~Hz}$, due in part to the choice of soft M1CS actuators and in part to increasing AO tip/tilt rejection at lower frequencies.

for two phases of the waveform that are $90^{\circ}$ apart (the temporal rms over one period of the sinusoidal waveform can be predicted from the response at these two phases). A quasi-static AO simulation is used to establish the spatial correctability of the pattern (using $\mathrm{MAOS}^{5}$ ). This spatial fitting error is generally small compared with the temporal fitting error from the limited bandwidth because the spatial pattern of M1 response is relatively smooth - Figure 2 illustrates this for a $30 \mathrm{~Hz}$ input, where the temporal correctability only reduces spatiallycorrectable motion by a factor of two. The length-scales of M1 motion are determined primarily by mirror cell dynamics, and are consistent with the length-scales of M1 segment motion inferred from AO DM data at Keck. ${ }^{6}$

The combination of soft voice-coil actuators for M1 and the lower AO-rejection bandwidth for tip/tilt means that the residual AO-corrected wavefront error is dominated by image jitter above roughly $10 \mathrm{~Hz}$; this is illustrated in Fig. 4. Furthermore, above roughly $10 \mathrm{~Hz}$, the image jitter is typically dominated by motion of M2. Overall telescope motion and M1 tip/tilt are important contributors to image jitter below $10 \mathrm{~Hz}$.

The combined AO-corrected wavefront error per Newton of force is shown for selected locations in Figure 5. A key observation is that the most sensitive frequency range for vibration forces is typically $5-20 \mathrm{~Hz}$. At lower frequencies, $\mathrm{AO}$ rejection is sufficiently good that the sensitivity decreases rapidly with decreasing frequency; there is also no dynamic amplification from telescope structural resonances below $5 \mathrm{~Hz}$. At sufficiently high frequency, inertial effects similarly result in a rapid decrease in sensitivity with increasing frequency. For simplicity, we thus (i) define a single sensitivity for each source location as the rms WFE per Newton over this $5-20 \mathrm{~Hz}$ band and (ii) define a frequency-dependent shaping filter that allows for higher forces at higher or lower frequencies. This shaping filter and average sensitivity are shown in Fig. 5, and the latter summarized for each source location in Table 1. Note that the rms WFE is extremely sensitive to several on-telescope locations in particular.

Forces in the facilities building or enclosure result in WFE only through the resulting pier motion, with some additional attenuation of vibration through the soil/pier. Based on data collected at Subaru Observatory, we conservatively estimate that a force in the facilities building results in a factor of at least 10 times less response than a force applied at the telescope pier, and a force at the enclosure pier results in a factor of 5 times less response (see Fig. 6 and [2]).

\section{VIBRATION BUDGET}

The vibration budget is intended to provide an initial force allocation to each subsystem or source of equipment vibration that helps ensure that the overall error budget allocation to equipment vibration is met. The vibration budget is constructed from the sensitivities estimated earlier and a subjective estimate of the level of difficulty of meeting force requirements for different sources. This is an initial allocation only, which will be revised as further information is available either from design or data. 

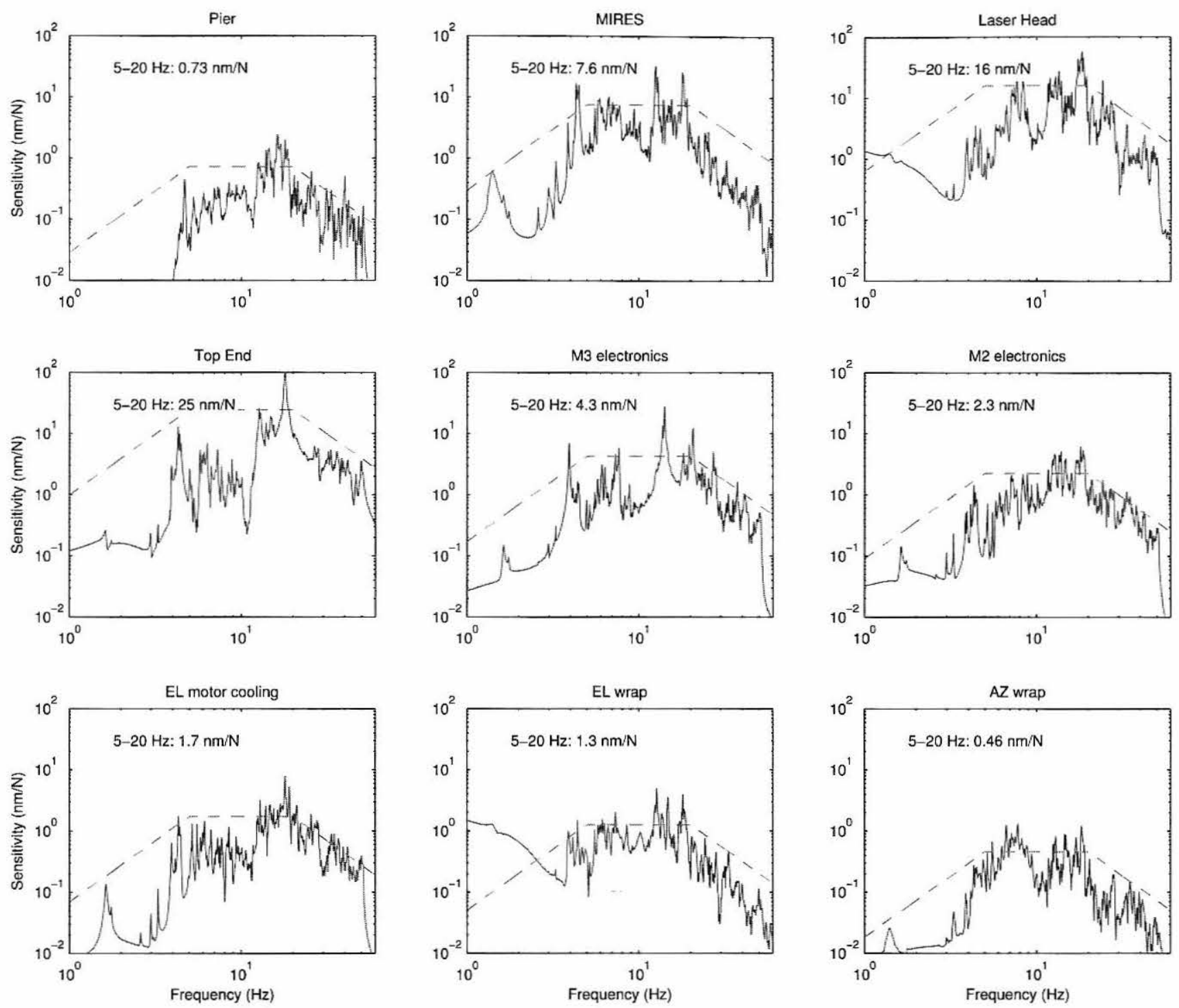

Figure 5. Sensitivity in $\mathrm{nm}$ of AO-corrected wavefront error per Newton of applied force as a function of frequency for forces at the locations given at the beginning of Section 2. For each location, the response is computed due to image motion; the contribution due to M1 segment motion is only included for selected locations. With the exception of the pier and cable wrap inputs, the combined wavefront error plotted is the rms over forces in $x, y$, and $z$ directions. Also shown is the "shaping filter" used to approximate the sensitivity, and the average sensitivity over the $5-20 \mathrm{~Hz}$ range.

Our initial allocation is shown in Table 2. For each source, this is the maximum allowable rms force, integrated over all frequencies after passing through the shaping filter described earlier that allows for higher forces at higher or lower frequencies. Note, individual subsystems can re-allocate between subcomponents within their subsystem.

While many of the force allocations appear to be exceptionally small, measurements of some representative equipment suggest that with isolation it may be possible to meet these requirements. For example, the cryopump tested in [2] results in less than $0.04 \mathrm{~N} \mathrm{rms}$ (after applying the shaping filter) when mounted on $3.6 \mathrm{~Hz}$ isolators.

It is clear that the vibration problem is dominated by components at a few locations with particularly high optical sensitivity, including anything at the telescope top-end near M2, the platform supporting the lasers (this may be amenable to redesign), and the Nasmyth platforms. Some large sources of vibration are located in the summit support building (e.g., chillers and pumps associated with the Summit Facilities subsystem, and HSB pumps associated with the telescope structure subsystem). However, because forces in these locations are significantly attenuated through the soil, relatively larger force allocations are allowable. 


\begin{tabular}{l|c} 
Source & Sensitivity $(\mathrm{nm} / \mathrm{N})$ \\
\hline Facilities Building* & 0.073 \\
Enclosure Pier* & 0.15 \\
Telescope Pier & 0.73 \\
Azimuth cable wrap & 0.46 \\
Elevation cable wrap & 1.3 \\
Instruments & 7.6 \\
Laser head & 16 \\
Laser, M2 electronics & 2.3 \\
Top end (LGSF, M2 cell) & 25 \\
M3, M1 electronics & 4.3 \\
Elevation-axis motor & 1.7 \\
\multicolumn{2}{c}{${ }^{*}$ estimated }
\end{tabular}

Table 1. Average sensitivity over $5-20 \mathrm{~Hz}$ for forces at the locations given in Section 2 , in nm of AO-corrected wavefront error per Newton of applied force (from Fig. 5). Sensitivities for sources in facilities building or enclosure pier are estimated as the sensitivity due to forces on the telescope pier, divided by a factor of 10 or 5 respectively (see Fig. 6).
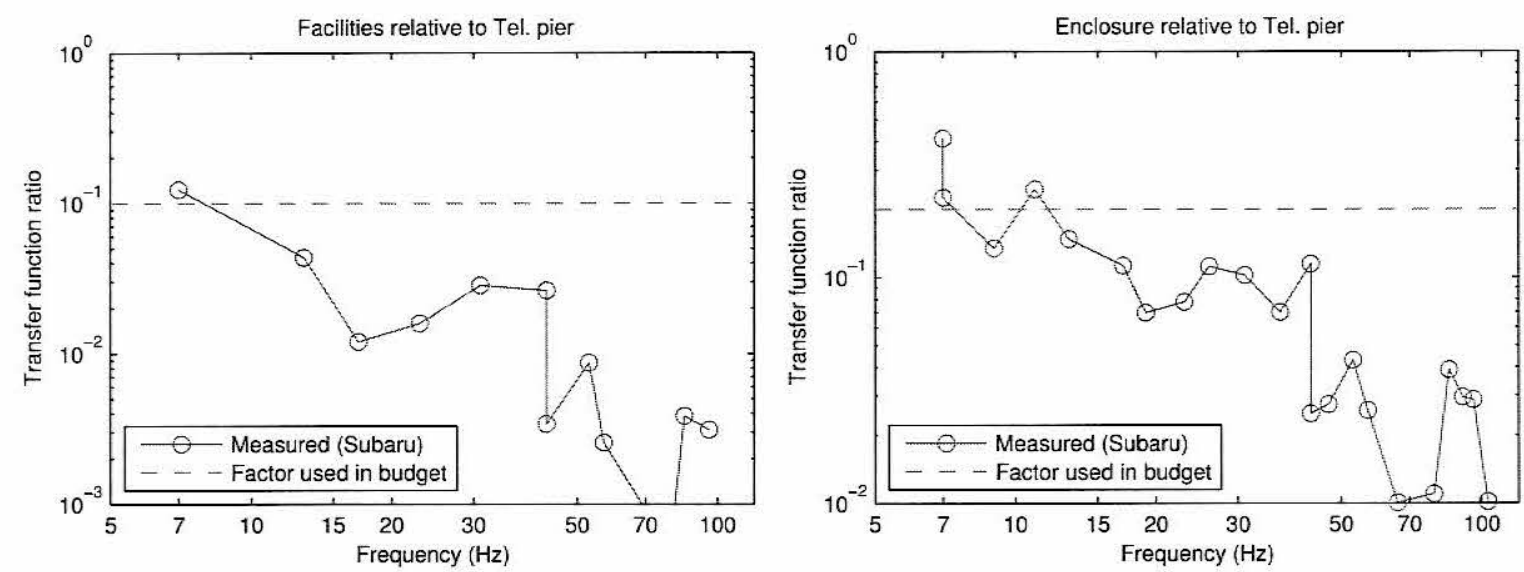

Figure 6. Ratio of vertical motion on telescope pier due to a force applied on the telescope pier compared with response at the same location due to a force applied in the facilities building (left) or enclosure pier (right). These are measured at Subaru Observatory (using reciprocity to obtain the transfer functions from remote locations). Reduction factors for horizontal motion are comparable. The reduction factors used in constructing the current TMT budget are likely to be conservative except at very low frequency (although the soil characteristics differ between the sites).

\section{CONCLUSIONS}

Image degradation due to equipment vibration has typically been addressed solely through "best practices" design choices, such as selecting low-vibration equipment, mounting equipment off-telescope where practical, and isolating large vibration sources. While these steps are clearly essential, TMT is establishing requirements on the allowable force levels at different locations in order to ensure that the AO-corrected wavefront errors induced by vibrating equipment remain at acceptable levels, and provide a framework for making cost-effective design choices. This vibration-budget is based on the modeled optical sensitivity resulting from vibrating forces at different locations and different frequencies.

There is clearly substantial uncertainty in predicting the sensitivity of rms WFE to forces as derived herein; the expected force levels from any particular source is also uncertain. Modeling the response of the telescope structure at the frequencies relevant for equipment vibration is challenging and not likely to be highly accurate. Furthermore, it is difficult to estimate unsteady forces from any equipment sources, particularly early in design. However, given the importance of vibration in AO-observations, it is essential that we make the best effort possible with existing tools. We hope that this effort will significantly increase the probability of delivering an observatory without significant vibration-related wavefront errors, and further, that this approach may be a model for other observatories in the future. 


\begin{tabular}{|c|c|c|c|c|c|}
\hline Subsystem & Subcomponent & $\begin{array}{l}\text { Sensitivity } \\
(\mathrm{nm} / \mathrm{N})\end{array}$ & $\begin{array}{l}\text { Allowable force } \\
\text { (N rms) }\end{array}$ & $\begin{array}{l}\text { Subcomponent } \\
\text { AO-WFE (nm) }\end{array}$ & $\begin{array}{c}\text { Aggregate } \\
\text { AO-WFE }(\mathrm{nm})\end{array}$ \\
\hline \multicolumn{2}{|c|}{ On Telescope } & & & & 24.7 \\
\hline Tel. Structure & & & & & 9.2 \\
\hline & Az Drive motor & 0.7 & 1.0 & 0.7 & \\
\hline & El Drive motor & 1.7 & 1.0 & 1.7 & \\
\hline & Az Cable wrap & 0.5 & 1.0 & 0.5 & \\
\hline & El Cable wrap & 1.3 & 1.0 & 1.3 & \\
\hline & HSB oil distribution & 0.7 & 1.0 & 0.7 & \\
\hline & Chilled water dist. & 7.6 & 1.0 & 7.6 & \\
\hline & Other & 4.6 & 1.0 & 4.6 & \\
\hline \multirow[t]{3}{*}{ M2 system } & & & & & 5.1 \\
\hline & M2 cell & 25 & 0.2 & 5.0 & \\
\hline & M2 electronics & 2.3 & 0.5 & 1.2 & \\
\hline M3 System & & 4.3 & 0.5 & 2.2 & 2.2 \\
\hline Engineering Sensors & & 25 & 0 & 0 & 0 \\
\hline \multirow{6}{*}{$\begin{array}{l}\text { Comm. \& Info. Systems } \\
\text { LGSF }\end{array}$} & & 7.6 & 1.0 & 7.6 & 7.6 \\
\hline & & & & & 9.5 \\
\hline & Top-end & 25.0 & 0.1 & 2.5 & \\
\hline & BTO & 7.6 & 0.5 & 3.8 & \\
\hline & Laser head & 16.0 & 0.5 & 8 & \\
\hline & Laser electronics & 2.3 & 1.0 & 2.3 & \\
\hline \multirow[t]{3}{*}{ Instrumentation cooling } & & & & & 10.7 \\
\hline & Cryocooling & 7.6 & 1.0 & 7.6 & \\
\hline & Refrigerant cooling & 7.6 & 1.0 & 7.6 & \\
\hline \multirow[t]{12}{*}{ Instruments } & & & & & 14.2 \\
\hline & APS & 7.6 & 0.5 & 3.8 & \\
\hline & NFIRAOS & 7.6 & 1.0 & 7.6 & \\
\hline & WFOS & 7.6 & 0.5 & 3.8 & \\
\hline & IRMS/MOSFIRE & 7.6 & 0.5 & 3.8 & \\
\hline & HROS & 7.6 & 0.5 & 3.8 & \\
\hline & IRMOS & 7.6 & 0.5 & 3.8 & \\
\hline & PFI & 7.6 & 0.5 & 3.8 & \\
\hline & MIRAO & 7.6 & 0.5 & 3.8 & \\
\hline & NIRES-B & 7.6 & 0.5 & 3.8 & \\
\hline & NIRES-R & 7.6 & 0.5 & 3.8 & \\
\hline & WIRC & 7.6 & 0.5 & 3.8 & \\
\hline \multicolumn{2}{|c|}{ Within Enclosure } & & & & 10.8 \\
\hline Enclosure & & 0.15 & 50 & 7.3 & \\
\hline Summit Facilities & & 0.7 & 10 & 7.3 & \\
\hline Tel. Structure & & 0.15 & 10 & 1.5 & \\
\hline Optical cleaning sys. & & 0.15 & 10 & 1.5 & \\
\hline Optical coating sys. & & 0.15 & 10 & 1.5 & \\
\hline Test instruments & & 0.15 & 1 & 0.15 & \\
\hline Optics handling eq. & & 0.15 & 10 & 1.5 & \\
\hline Test instrument ctrl & & 0.15 & 1 & 0.15 & \\
\hline Observatory safety sys. & & 0.15 & 1 & 0.15 & \\
\hline Engineering sensors & & 0.15 & 10 & 1.5 & \\
\hline Comm. \& Info. sys & & 0.15 & 1 & 0.15 & \\
\hline \multicolumn{2}{|c|}{ Inside Support Building } & & & & 8.8 \\
\hline Summit facilities & & 0.07 & 100 & 7.3 & \\
\hline Telescope structure & & 0.07 & 50 & 3.7 & \\
\hline Miscellaneous & & 0.07 & 44 & 3.2 & \\
\hline
\end{tabular}

Table 2. Preliminary vibration budget, giving the allowable force level for each source, and the estimated sensitivity (Table 1) and corresponding AO-corrected wavefront error; the aggregate wavefront error from each subsystem is also given. Many subsystems have equipment in the support building; for clarity these are grouped here under "miscellaneous". 


\section{Acknowledgments}

The TMT Project gratefully acknowledges the support of the TMT collaborating institutions. They are the Association of Canadian Universities for Research in Astronomy (ACURA), the California Institute of Technology, the University of California, the National Astronomical Observatory of Japan, the National Astronomical Observatories of China and their consortium partners, and the Department of Science and Technology of India and their supported institutes. This work was supported as well by the Gordon and Betty Moore Foundation, the Canada Foundation for Innovation, the Ontario Ministry of Research and Innovation, the National Research Council of Canada, the Natural Sciences and Engineering Research Council of Canada, the British Columbia Knowledge Development Fund, the Association of Universities for Research in Astronomy (AURA), the U.S. National Science Foundation and the National Institutes of Natural Sciences of Japan.

\section{REFERENCES}

[1] Kulcsár, C. et al., "Vibrations in AO control: a short analysis of on-sky data around the world," Proc. SPIE 8447, Adaptive Optics Systems III, 2012.

[2] Thompson, H., MacMartin, D. G., Byrnes, P., Tomono, D., and Terada, H., "Measuring transmission and forces from observatory equipment vibration," Proc. SPIE 9145, 2014.

[3] MacMartin, D. G. and Thompson, H., "Development of vibration source requirements for TMT to ensure AO performance," AO4ELT3, 2013.

[4] MacMartin, D. G., Thompson, P., Colavita, M. M., and Sirota, M. J., "Dynamic analysis of the active-controlled segmented mirror of the Thirty Meter Telescope," IEEE Trans. Control Sys. Tech., Vol. 22, No. 1, pp. 58-68, 2013.

[5] Wang, L. and Ellerbroek, B., "Computer simulations and real-time control of ELT AO systems using graphical processing units," Proc. SPIE 8447, 2012.

[6] MacMynowski, D. G., Colavita, M. M., Skidmore, W., and Vogiatzis, K., "Primary mirror dynamic disturbance models for TMT: Vibration and wind," Proc. SPIE 7738, 2010. 